\title{
Averaging High Redshift X-ray and $\gamma$-ray Spectra of Faint Sources
}

\author{
Tahir Yaqoob $^{1} \dagger$ \\ ${ }^{1}$ Department of Physics \& Astronomy, Johns Hopkins University, Baltimore, MD 21212, USA.
}

\begin{abstract}
Population studies of faint X-ray sources (e.g. galaxies, AGN, $\gamma$-ray bursts and their afterglows) have become more and more common. The problem of obtaining an average spectrum in the rest-frame of the sources is non-trivial. We show that conventional methods for averaging low signal-to-noise X-ray spectra, when applied to sources at different redshifts, result in a mean rest-frame spectrum that can exhibit artificial features. These include the broadening and weakening of emission/absorption lines, a broad dip in the continuum above an emission line, and a spectral hardening at the highest energies. All of these effects have been observed in real data and have been given fairly weighty astrophysical and/or cosmological interpretations. We present a new method of averaging which considerably reduces the severity of the problems.
\end{abstract}

Keywords. methods: data analysis, X-rays: galaxies, cosmology: observations.

\section{Principal Problems}

While the spectral averaging problem has been discussed and studied in detail in the literature for sources with $z \sim 0$ (e.g. Yaqoob et al. 2002; Lubiński 2004), there are problems with weak sources at different redshifts. Firstly, unless the detector response is nearly diagonal, "unfolding" the counts spectra of individual sources before blueshifting to the rest-frame is unavoidable. One is then forced to work with "data" which may exhibit model-dependence. Secondly, if the individual spectra have too few counts per bin, one is forced to group the spectral channels in such a way that there is a minimum number of photon counts per channel in order that the background can be subtracted and Gaussian statistics used to calculate and propagate the statistical errors. Grouping the spectral channels can introduce distortion which cannot be reversed. Moreover, the conventional approach is to group the background spectra using the same channel groups as the on-source spectra but this does not guarantee a sufficient number of counts per bin in the background spectra. Note that accumulating a much higher signal-to-noise background spectrum does not avoid the grouping problem because the individual onsource spectra may still be in the Poisson regime and each spectrum may simply be an under-sampled version of what one is trying to measure (the true source spectra, which would be obtained with an infinitely long exposure time).

\section{Simulation Results and a New Method}

We performed simulations of 200 sources with redshifts uniformly and randomly distributed between 0.5 and 2.5 , and $2-10 \mathrm{keV}$ fluxes uniformly and randomly distributed in the range $6-60 \times 10^{-14} \mathrm{ergs}^{-2} \mathrm{sm}^{-1}$ and constructed mean rest-frame spectra with and without spectral grouping and with and without background subtraction, and with

$\dagger$ Also: Exploration of the Universe Division, NASA/Goddard Space Flight Center, Greenbelt, MD 20771, USA. 

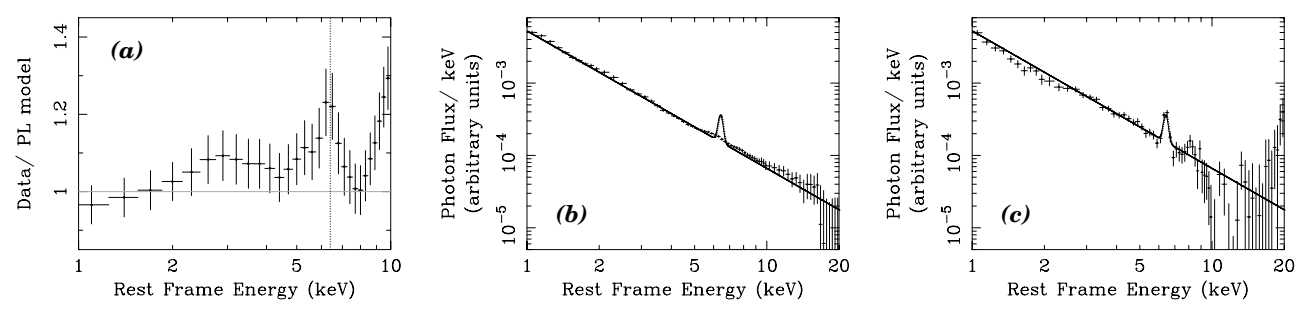

Figure 1. Mean rest-frame spectra from $20 \mathrm{ks}$ exposure simulations of a power law plus unresolved narrow line (see text). (a) Conventional methods: no background; spectral channels grouped. Ratio to a simple to power law only. (b) Conventional methods: simulated spectra background-subtracted and grouped. (c) New method: background-subtraction, but no spectral grouping. Narrow line is recovered, continuum distortion reduced.

various exposure times. All sources had the same input spectrum, namely a power law with a photon index of 1.9, and a narrow (unresolved) Gaussian Fe K emission line, with a rest-frame energy and Gaussian width of $6.4 \mathrm{keV}$ and $0.005 \mathrm{keV}$ respectively. We used $X M M-N e w t o n$ pn (CCD) detector responses. Here we only briefly summarize the results of our findings.

Fig. 1 shows the results when the exposure time of each spectrum was $20 \mathrm{ks}$. With no background simulated, but using conventional methods of grouping, unfolding, and blueshifting, Fig. 1(a) shows the result of fitting the mean rest-frame spectrum with a simple power law only. Considerable distortion is apparent. Fig. 1(b) shows the mean restframe spectrum obtained using conventional methods, this time including simulation and subtraction of background. Again, the input spectrum is not recovered well. These kind of spectral distortions (broadening of the emission line and continuum hardening) have been observed using real data (e.g. Streblyanska 2004, 2005 and references therein). We present a new method in which the background spectra are grouped, the on-source are not grouped, and the background subtraction is done in the rest-frame. Thus, the on-source spectra and background spectra are separately unfolded and blueshifted, separately coadded, and only then (in the rest-frame) is the (mean) background subtracted from the on-source (mean) spectrum. Fig. 1(c) shows that the method is able to recover the input spectrum much better than the conventional methods. At first it may seem odd that the background spectra are unfolded and blueshifted since the background is local (i.e. at $z \sim 0$ ) and may not even have passed through the telescope. However, with conventional methods, in which the background-subtracted spectra are unfolded and blueshifted, the background spectra $A R E$ implicitly unfolded and blueshifted.

\section{Conclusions}

We have shown how to construct mean rest-frame spectra of faint X-ray sources at different redshifts. Conventional methods suffer more from artificial broadening of emission and absorption lines, spectral hardening and other continuum distortion.

\section{References}

Lubiński, P. 2004, MNRAS 350, 596

Streblyanska, A., Bergeron, J., Brunner, H., Finoguenov, A., Hasinger, G., \& Mainieri, V. 2004, NuPhS 132, 232

Streblyanska, A., Hasinger, G., Finoguenov, A., Barcons, X., Mateos, S., \& Fabian, A. C. 2005, A $\& A 432,395$

Yaqoob, T., Padmanabhan, U., Dotani, T., \& Nandra, K. 2002, ApJ, 569, 487 